\title{
Au nanoparticles in PMMA matrix: In situ synthesis and the effect of $\mathrm{Au}$ nanoparticles on PMMA conductivity
}

\author{
Eda Yilmaz, Sefik Suzer* \\ Bilkent University, Chemistry Department, 06800 Ankara, Turkey
}

\section{A R T I C L E I N F O}

\section{Article history:}

Received 29 January 2010

Received in revised form 9 April 2010

Accepted 17 April 2010

Available online 27 April 2010

\section{Keywords:}

Au-nanoparticles

PMMA matrix

In situ synthesis

Charging

\begin{abstract}
A B S T R A C T
Thin PMMA films with and without gold nanoparticles were subjected to $\pm 10 \mathrm{~V}$ d.c. and a.c. (square wave) excitations in various frequencies while recording their XPS spectra, and the resulting differences due to charging were examined. Both pure PMMA films and films containing gold nanoparticles showed charging shifts, but those of pure PMMA were more extensive than of PMMA containing gold nanoparticles, suggesting enhanced conductivity, induced by the incorporated gold nanoparticles. Non-charging behavior for these films was also observed with the increase of gold nanoparticle concentration. Gold nanoparticles were in situ synthesized and photo-patterned within the polymer films by UV irradiation.
\end{abstract}

(C) 2010 Elsevier B.V. All rights reserved.

\section{Introduction}

Polymer-nanoparticle composites are important candidates for new age technological devices and ongoing research by many different groups is performed for production and characterization of these systems [1-8]. Nanoparticles (NPs) may be synthesized before integration to polymeric matrix or within them (in situ). Both methods may have their advantages and disadvantages depending on the usage and many examples for both are available in literature [9-12]. An efficient approach, used for the production of nanoparticles within the polymer matrix is employment of irradiation. Energetic light is widely employed for reduction of metal salts as it can be used in solution phase as well as for solid state synthesis $[13,14]$. Korchev et al. proposed that silver nanoparticles can be produced in SPEEK/PVA films by direct irradiation with $350 \mathrm{~nm}$ UV light [15]. Alexandrov et al. used UV light to initiate gold nanoparticle growth in PMMA films and finalized the synthesis by annealing the samples at a higher temperature [16]. A recent study has reported on a model for the growth kinetics of the nanoparticles within polymeric matrices [17]. In our method of synthesis of gold NPs in PMMA matrices, we also employ UV radiation to reduce gold ions to gold atoms and consequently gold atoms aggregate and form gold NPs within the polymer matrix at room temperature [18].

UV-vis spectrometry is a commonly used technique to detect/follow formation and/or presence of nanoparticles, since,

\footnotetext{
* Corresponding author. Tel.: +90 312 2901476; fax: +90 3122664068.

E-mail address: suzer@fen.bilkent.edu.tr (S. Suzer).
}

noble metal NPs like gold and silver, have distinct absorption peaks of their surface plasmon resonance in the visible region. X-ray photoelectron spectrometry (XPS) is another instrument widely utilized to obtain information about NPs and their composites with polymer films, while special care has to be exercised to overcome charging problems. In XPS measurements, a current is generated by the photoelectrons and secondary electrons from the sample and these cause charging for non-conducting samples, since they can not easily neutralize the generated positive charges. In early years of XPS experiments, the charging of the organic polymer films had been considered as a nuisance and many successful techniques were developed to overcome this problem. Indeed charging causes shifts in the binding energies of the peaks, and inconsistent results might be produced. But it is also proved to be true that when used correctly, additional information about the samples can be harvested using charging as a tool $[19,20]$. Furthermore, applying an external voltage bias to the sample during XPS measurements causes shifts in the positions depending on the polarity, as well as the nature of the sample. For conducting samples the peak positions shift as much as the applied bias, but for non-conducting samples the shift is observed to be less and nonlinear due to charging, which has been successfully used to differentiate between the fiber and the matrix signals of a carbon composite material, and/or other surface heterostructures [21-23]. This nonlinear behavior contains additional molecular and structural information, as was recently reviewed by Sezen et al. [24] through impedance-like XPS measurements on PMMA, PS (polystyrene), and their blended composite films. The main aim of the present study is to report similar XPS measurements performed on PMMA and Au NP/PMMA films while 


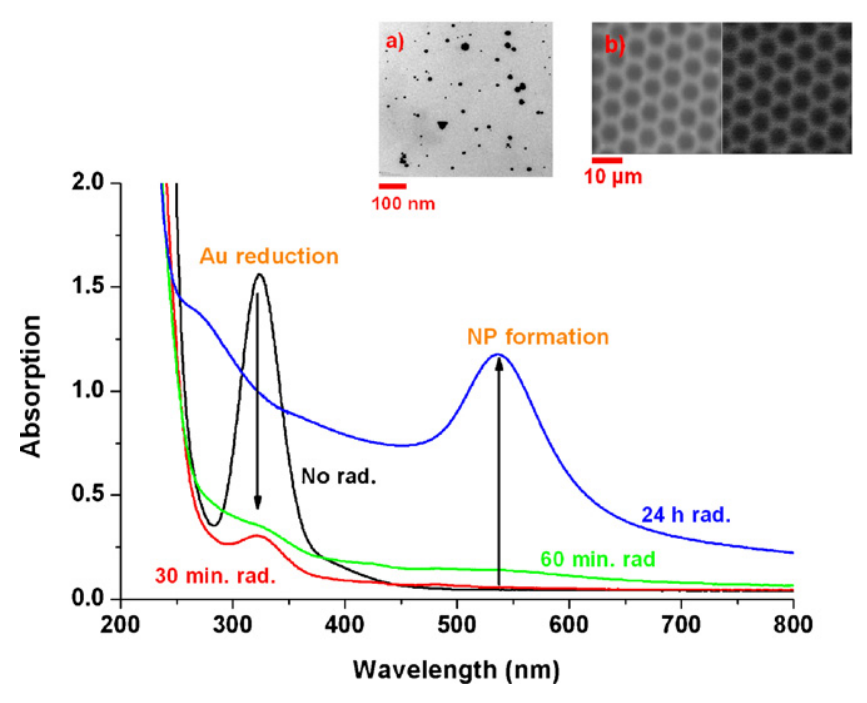

Fig. 1. UV spectra of PMMA film showing $\mathrm{Au}^{3+}$ reduction and Au NP formation during irradiation. Inset (a) shows the TEM image and (b) shows the SEM images of the photopatterned PMMA film with Au NPs. (Left side: secondary electron image of the film, right side: backscattered electron image of the film.)

applying external bias to these samples. This method enables us to demonstrate the profound effect of gold NPs on the conductivity of PMMA films, not easily measurable using standard electrical measurement techniques.

\section{Experimental}

Tetrachloroauric $\left(\mathrm{HAuCl}_{4}\right)$ and polymethylmethacrylate (PMMA) were purchased from Aldrich and used without further purification. Solutions containing $1 \mathrm{mM} \mathrm{HAuCl}_{4}$ and $0.5 \%(\mathrm{w} / \mathrm{v})$ PMMA in acetone were used to prepare the films by spin coating on $\mathrm{Si}$ or quartz wafers. Si wafers were first treated with HF to remove the native oxide layer prior to use. Thicker films were also prepared for UV-vis measurements with more concentrated PMMA solutions by room temperature evaporation in saturated acetone atmosphere. The resulting films were reduced with $254 \mathrm{~nm}$ wavelength UV light for varying durations ranging from 1 to $24 \mathrm{~h}$. Reduction of $\mathrm{Au}^{3+}$ ions and formation of $\mathrm{Au}^{0}$ nanoparticles were followed by a Cary 5E UV-vis-NIR spectrometer. SEM images were taken by Carl-Zeiss EVO-60 SEM microscope, and an FEI-Tecnai G2F30 was used for TEM images. A Kratos ES300 spectrometer with a $\mathrm{Mg} \mathrm{K} \alpha$ (non-monochromatized) source at $1253.6 \mathrm{eV}$ was used to record the XPS spectra. Charging experiments were performed by applying d.c. or square wave pulses of $\pm 10 \mathrm{~V}$ amplitude with different frequencies to the sample. XPS peaks were fitted using the XPSPEAK4.0 fitting program [25]. The data were recorded at a $90^{\circ}$ take-off angle.

\section{Results and discussion}

The synthesis of Au nanoparticles by the in situ UV irradiation within the PMMA matrix and patterning can be easily monitored by UV-vis spectrometer step by step as depicted in Fig. 1. At the beginning, the films contain gold ions represented by the peak around $320 \mathrm{~nm}$. After $30 \mathrm{~min}$ of irradiation of the film this peak disappears, indicating that the reduction of gold ions to gold atoms is completed. In the spectrum taken after $1 \mathrm{~h}$ of irradiation, the gold NP's plasmon resonance band starts to appear, and grows in time. Formation of gold NPs is completed within 12-24h depending on the concentration of gold. As can be gathered from the TEM image shown in the inset (a), particles with varying shapes and sizes $(5-50 \mathrm{~nm})$ are formed. One of the advantages of synthesizing gold
NPs within polymer films is the feasibility of easy photo-patterning. Films having patterns made of regions with and without gold NPs can be produced by using masks designed to cut off the UV radiation at desired places as shown in the inset (b) of Fig. 1, which displays both the secondary and the backscattered electron SEM images of the photo-patterned film. The bright areas contain gold nanoparticles and they were irradiated by UV light, whereas the dark areas were under the mask so were not exposed to radiation. The size of the mask can be varied according to the application and satisfactory contrast can be gained in the micron range.

Characterization of PMMA films containing gold NPs is the main aspect of our present study for better understanding of the polymer-nanoparticle interactions towards advanced applications of these composite systems. Normally, PMMA is used as a dielectric component in electronic applications due to its high resistance [26]. On the other hand, possibility to controllably change the conductivity of PMMA by addition of conducting species is also of concern [27]. For this purpose Abyaneh et al. [28]. reported the decrease in the resistance of PMMA films with increasing gold NP content. Also in an another recent study, the increased conductivity of PMMA by gold ions was studied by Salvadori et al. [29] with a buried conducting layer of metal/polymer nanocomposite which was formed by very low energy gold ion implementation, where they determined a relatively high percolation dose of $1 \times 10^{16}$ atoms $/ \mathrm{cm}^{2}$.

We present a new method to investigate the conductivity of gold nanoparticle-PMMA composites using XPS, focusing on the charging properties of PMMA and Au NPs/PMMA films to understand the effect of Au NPs on the electrical properties of PMMA films. Using charging as a tool to investigate the resistance of PMMA films in XPS has its own advantages. The commonly used method of measuring resistance of polymeric films, the $I-V$ measurement, are problematic since the resistances are usually very high and good electrical contacts are not easily obtained. On the other hand, use of XPS for harvesting electrical properties is a non-contact technique has been shown to be feasible and carried out down to sub-micron sizes [30-32].

As discussed earlier, applying voltage to the sample during XPS acquisition effects the position of the lines and additional information about samples can be derived from this property. For example, when $\pm 10 \mathrm{~V}$ is applied to the sample, if the sample is conductive a direct shift of $10.0 \mathrm{eV}$ of the line positions is observed. But if the sample is nonconductive, shifts smaller than $10.0 \mathrm{eV}$ are observed [21-24]. Our experimental setup for our XPS measurements is given in the inset of Fig. 2. In the same figure, $C$ 1s spectra of the PMMA and Au NP/PMMA films, grounded and subjected to $\pm 10 \mathrm{~V}$ potentials are given. Both films were irradiated for $12 \mathrm{~h}$ to have identical conditions except for the presence of gold NPs. As can be seen from the figure, the binding energy difference between $C 1 \mathrm{~s}$ peaks at -10 and $+10 \mathrm{~V}$ potentials is about $11.3 \mathrm{eV}$ for PMMA film while it is $16.0 \mathrm{eV}$ for Au NP/PMMA films. This $4.7 \mathrm{eV}$ difference of charging between these two samples suggests that the drastic charging of the PMMA-only film is significantly reduced by the addition of the Au NPs. As shown at the bottom of the same figure, in the film containing ca. 2 times (10\% Au by weight) the charging behavior is completely diminished as evidenced by the measured binding energy difference between $-10 \mathrm{~V}$ and $+10 \mathrm{~V}$ potentials to be nearly $20.0 \mathrm{eV}$, compared to the less concentrated film which has a charging difference of $16.0 \mathrm{eV}$ under the same conditions.

Dynamics of the charging behavior can also be investigated by applying square wave (SQW) potentials of different frequencies. When the XPS spectra of $O 1 \mathrm{~s}$ and $C 1 \mathrm{~s}$ peaks of PMMA films are measured by applying $\pm 10 \mathrm{~V}$ square wave voltage with different frequencies, it is observed that at high frequencies, since the applied voltage changes in between $-10 \mathrm{~V}$ and $+10 \mathrm{~V}$ quickly, the film does not have enough time to charge and discharge, and the difference between the separated peaks is $20.0 \mathrm{eV}$. But at low frequencies, 

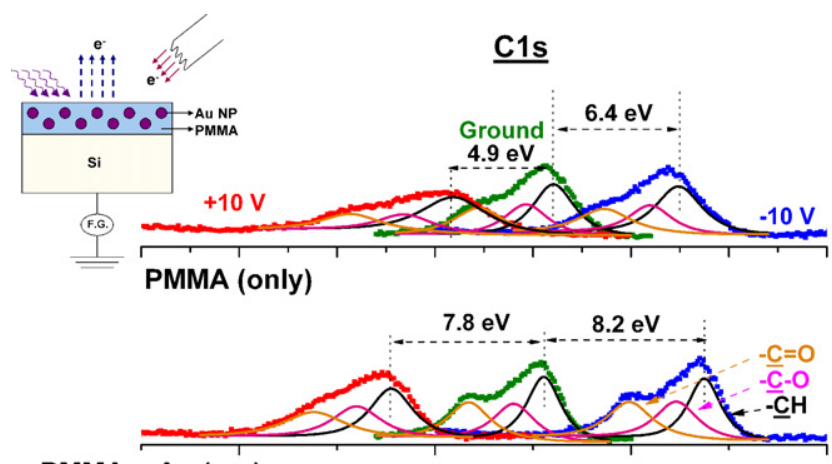

PMMA + Au (n.p)

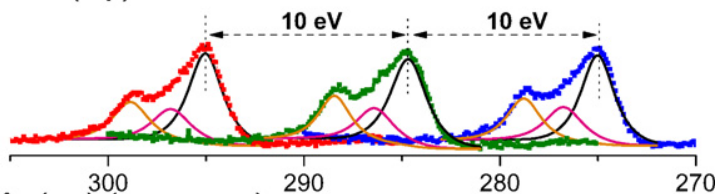

PMMA + Au (n.p) (more conc.)

Fig. 2. C 1s spectra of PMMA, Au/PMMA and more concentrated Au/PMMA films grounded and subjected to $\pm 10 \mathrm{~V}$ potentials.

since ample time is allowed for the samples to charge/discharge, the difference is significantly lower than $20.0 \mathrm{eV}$, as shown in Fig. 3. Also at high frequencies, although we do not observe less than $20.0 \mathrm{eV}$ separation between the twinned peaks, the charging manifests as an off-set in the positions of the peaks. In charging samples this off-set in the positions is always observed, which increases as for example with the thickness of the films [16,24]. In Fig. 4, we display the response of three films to the SQW excitation at $10 \mathrm{~Hz}$, which is considered to be a relatively high frequency, together with their grounded spectra. In the conducting graphite film and at $10 \mathrm{~Hz}$, the $C 1 \mathrm{~s}$ peak is twinned at exactly +10.0 and $-10.0 \mathrm{eV}$, from its grounded position, but both for PMMA only and $\mathrm{Au}(\mathrm{NP}) / \mathrm{PMMA}$ films the $C 1 \mathrm{~s}$ peaks are off-set by 1.3 and $0.3 \mathrm{eV}$, respectively, which is another experimental evidence of the charging behavior of these films [16]. We must also note that, as depicted in Fig. 5, using conventional 4 probe electrical measurements we are not able to detect any significant change in the bulk conductivity of the films after incorporation of the Au NPs even in the case of the ones containing $10 \%$ Au by weight. Hence, the electrical property that we are probing with our modified XPS measurements reflect truly surface properties of these films.

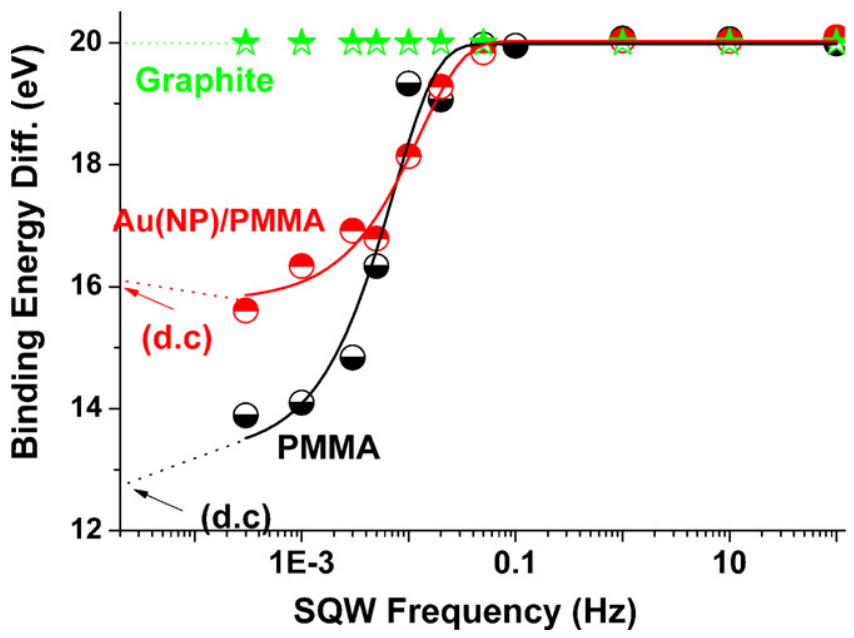

Fig. 3. The measured binding energy difference between the twinned $C 1$ s peaks, plotted against the logarithm of the frequency of the $\pm 10 \mathrm{~V}$ SQW excitation.

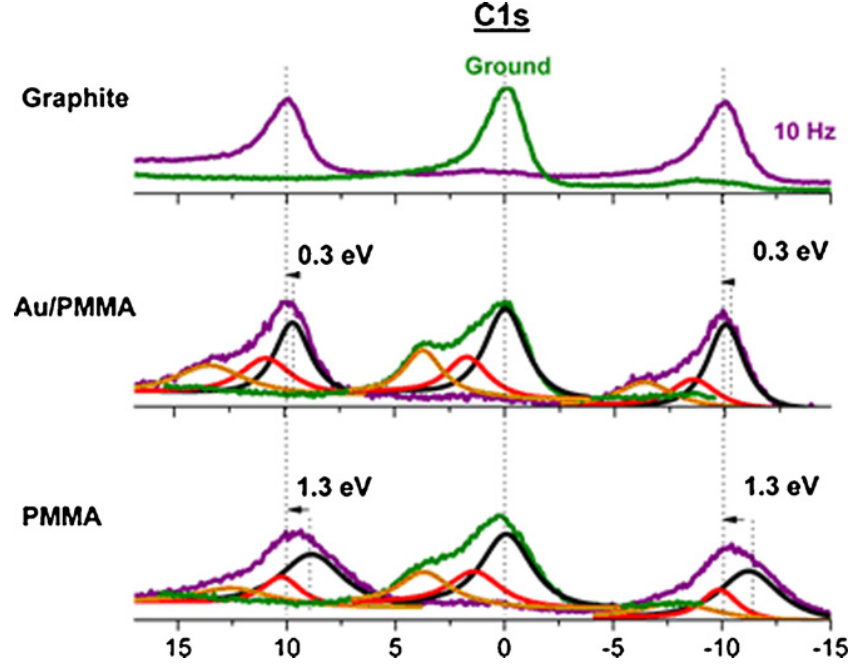

Fig. 4. The $C 1 \mathrm{~s}$ spectra of graphite, PMMA and Au NP/PMMA films under $\pm 10 \mathrm{~Hz}$ SQW excitation.

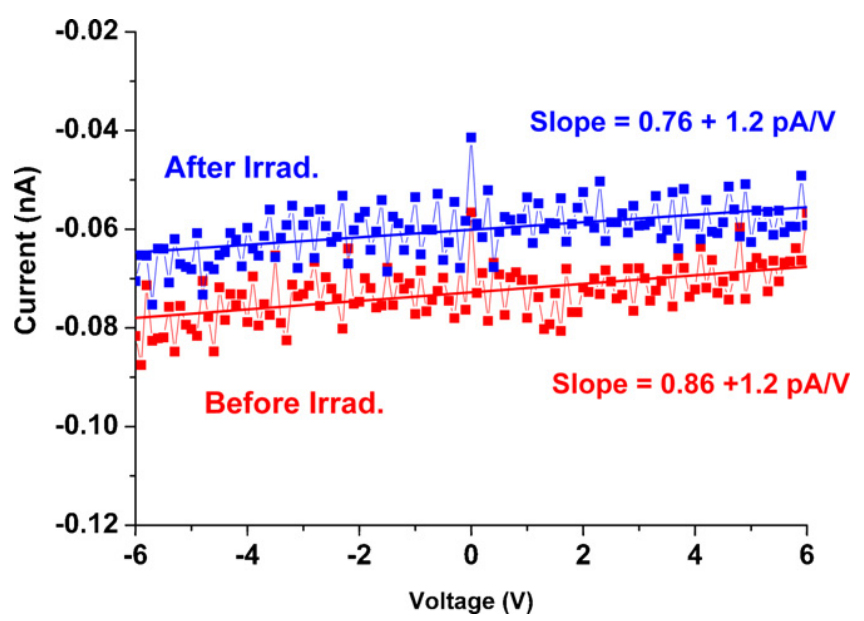

Fig. 5. $I-V$ curves of the AuNP/PMMA film before and after irradiation.

\section{Conclusions}

In summary, in this work, the effect of gold NPs to the conductivity of PMMA films was investigated with XPS measurements by applying external bias to the films. It was shown that the incorporation of gold NPs to PMMA films increase the surface conductivity of the films, resulting in less charging on the surface. This simple yet powerful method requires less effort for the sample preparation and yields reliable results even in low concentrations of incorporated metallic NPs.

\section{Acknowledgements}

This work was partially supported by TUBA (Turkish Academy of Sciences), TUBITAK (The Scientific and Technological Research Council of Turkey) through the Grant No. 106T409, and by the European Union 7. Framework Project Unam-Regpot, Grant No. 203953.

\section{References}

[1] M.C. Daniel, D. Astruc, Gold nanoparticles: assembly, supramolecular chemistry, quantum-size-related properties, and applications toward biology, catalysis, and nanotechnology, Chem. Rev. 104 (1) (2004) 293-346.

[2] A.N. Shipway, E. Katz, I. Willner, Nanoparticle arrays on surfaces for electronic optical, and sensor applications, ChemPhysChem 1 (1) (2000) 18-52; 
G.A Gaddy, J.L. McLain, A.S. Korchev, et al., Kinetics of silver particle photogeneration in crosslinked PVA/PAA films, J. Phys. Chem. B 108 (39) (2004) $14858-14865$.

[3] T. Tanaka, K. Yamaguchi, S. Yamamoto, Rhodamine-B-doped and Au(III)-doped PMMA film for three-dimensional multi-layered optical memory, Opt. Commun. 212 (1-3) (2002) 45-50.

[4] C.P.E. Wong, Polymers for Electronic and Photonic Applications, Academic Press, Boston, 1993.

[5] V. Zaporojtchenko, T. Strunskus, K. Behnke, et al., Metal/polymer interfaces with designed morphologies, J. Adhes. Sci. Technol. 14 (3) (2000) 467-490.

[6] V. Zaporojtchenko, T. Strunskus, K. Behnke, et al., Formation of metal-polymer interfaces by metal evaporation: influence of deposition parameters and defects, Microelectron. Eng. 50 (1-4) (2000) 465-471.

[7] V. Zaporojtchenko, J. Zekonyte, A. Biswas, et al., Controlled growth of nanosize metal clusters on polymers by using VPD method, Surf. Sci. 532 (2003) 300-305.

[8] V. Zaporojtchenko, J. Zekonyte, S. Wille, et al., Tailoring of the PS surface with low energy ions: relevance to growth and adhesion of noble metals, Nucl. Instrum. Methods Phys. Res. Sect. B-Beam Interact. Mater. Atoms 236 (2005) 95-102.

[9] F. Vitale, L. Mirenghi, E. Piscopiello, et al., Gold nanoclusters-organometallic polymer nanocomposites: synthesis and characterization, Mater. Sci. Eng. CBiomimetic Supramol. Syst. 27 (5-8) (2007) 1300-1304.

[10] S. Horiuchi, M.I. Sarwar, Y. Nakao, Nanoscale assembly of metal clusters in block copolymer films with vapor of a metal-acetylacetonato complex using a dry process, Adv. Mater. 12 (20) (2000) 1507-1511.

[11] S. Asakura, A. Hozumi, T. Yamaguchi, A. Fuwa, A simple lithographic method employing $172 \mathrm{~nm}$ vacuum ultraviolet light to prepare positive- and negativetone poly(methyly methacrylate) patters, Thin Solid Films 500 (2006) 237-240.

[12] H. Kim, H.-B.-R. Lee, W.-J. Maeng, Applications of atomic layer deposition and emerging nanodevices, Thin Solid Films 517 (2009) 2563-2580.

[13] E. Gachard, H. Remita, J. Khatouri, et al., Radiation-induced and chemical formation of gold clusters, New J. Chem. 22 (11) (1998) 1257-1265.

[14] F. Karadas, G. Ertas, E. Ozkaraoglu, et al., X-ray-induced production of gold nanoparticles on $\mathrm{SiO}_{2} / \mathrm{Si}$ system and in a poly(methyl methacrylate) matrix Langmuir 21 (1) (2005) 437-442.

[15] A.S. Korchev, T. Konovalova, V. Cammarata, et al., Radical-induced generation of small silver particles in SPEEK/PVA polymer films and solutions: UV-vis, EPR, and FT-IR studies, Langmuir 22 (1) (2006) 375-384.

[16] A Alexandrov, L. Smirnova, N. Yakimovich, et al., UV-initiated growth of gold nanoparticles in PMMA matrix, Appl. Surf. Sci. 248 (1-4) (2005) 181-184.
[17] N. Sapogova, N. Bityurin, Model for UV induced formation of gold nanoparticles in solid polymeric matrices, Appl. Surf. Sci. 255 (2009) 9613-9616.

[18] E. Ozkaraoglu, K. Tunc, S. Suzer, Preparation of Au and Au-Pt nanoparticles within PMMA matrix using UV and X-ray irradiation, Polymer 50 (2) (2009) 462-466.

[19] P.M.A. Sherwood, Exploiting differential sample charging in X-ray photoelectron spectroscopy, Surf. Sci. 600 (2006) 771-772.

[20] P.M.A. Sherwood, Historical perspectives on charging issues in XPS, J. Electron. Spectrosc. Relat. Phenom. 176 (2010) 2-4.

[21] J.D. Miller, W.C. Harris, G.W. Zajac, Composite interface analysis using voltage contrast XPS, Surf. Interface Anal. 20 (12) (1993) 977-983.

[22] M.A. Rooke, P.M.A. Sherwood, X-ray photoelectron spectroscopic study of ion etching and electrical biasing of silicon-nitride on a carbon-fiber, Surf. Interface Anal. 21 (10) (1994) 681-690.

[23] N.J. Havercroft, P.M.A. Sherwood, Use of differential surface charging to separate chemical differences in X-ray photoelectron spectroscopy, Surf. Interface Anal. 29 (3) (2000) 232-240.

[24] H. Sezen, G. Ertas, S. Suzer, Methods for probing charging properties of polymeric materials using XPS, J. Electron. Spectrosc. Relat. Phenom. 178-179 (2010) 373-379.

[25] R.W.M. Kwok, Available at http://phy.cuhk.edu.hk/ surface/XPSPEAK.

[26] W.H. Teh, C.T. Liang, M. Graham, et al., Cross-linked PMMA as a lowdimensional dielectric sacrificial layer, J. Microelectromech. Syst. 12 (5) (2003) 641-648.

[27] V. Raja, A.K. Sharma, V. Rao, Impedance spectroscopic and dielectric analysis of PMMA-CO-P4VPNO polymer films, Mater. Lett. 58 (26) (2004) 3242-3247; W. Zheng, S.C. Wong, Electrical conductivity and dielectric properties of PMMA/expanded graphite composites, Compos. Sci. Technol. 63 (2) (2003) 225-235.

[28] M.K. Abyaneh, R. Pasricha, S.W. Gosavi, et al., Thermally assisted semiconductor-like to insulator transition in gold-poly(methyl methacrylate) nanocomposites, Nanotechnology 17 (16) (2006) 4129-4134.

[29] M.C. Salvadori, M. Cattani, F.S. Teixeira, et al., Conducting polymer formed by low energy gold ion implantation, Appl. Phys. Lett. 93 (7) (2008).

[30] H. Cohen, Chemically resolved electrical measurements using X-ray photoelectron spectroscopy, Appl. Phys. Lett. 85 (7) (2004) 1271-1273.

[31] H. Cohen, R. Maoz, J. Sagiv, Transient charge accumulation in a capacitive selfassembled monolayer, Nano Lett. 6 (11) (2006) 2462-2466.

[32] G. Ertas, U.K. Demirok, A. Atalar, et al., X-ray photoelectron spectroscopy for resistance-capacitance measurements of surface structures, Appl. Phys. Lett. 86 (18) (2005) 83110. 